\title{
El qué, por qué, cómo y cuándo de la tutoría: desarrollo de la ayuda y las habilidades de tutoría en niños
}

-Segunda parte-

David Wood*

\author{
Publicado originalmente en \\ Literacy Teaching and Learning: An International \\ Journal of Early Reading and Writing. \\ Vol. 7 (pp. I-30). Copyright 2003. \\ Reprinted with permission from \\ the Reading Recovery Council of North America.
}

Traducido por:

Luis Alejandro Andrade Lotero

María Fernanda Cobo Charry

Presentación de la segunda parte del artículo del profesor David Wood

El profesor David Wood, psicólogo inglés, es conocido por sus aportes a la psicología del aprendizaje y por su famoso artículo

Centre for Research in Development, Instruction, and Training (CREDIT), University of Nottingham 
en colaboración con Jerome Bruner y G. Ross, el cual introdujo por primera vez el término "andamiaje" (ver la introducción a la primera parte del artículo). El profesor Wood también es conocido por sus estudios acerca de la Teoría de la Contingencia. Esta teoría tiene como componentes principales los conceptos de tutoría, aprendizaje y andamiaje.

El concepto de andamiaje hace referencia a la ayuda que un sujeto más capaz le brinda a un aprendiz, pero que paulatinamente debe ser retirada y desmontada luego de haber construido las nuevas estructuras de pensamiento. El concepto de tutoría se refiere a una relación muy particular que se crea entre un tutor y un aprendiz. El tutor es responsable de crear una Zona de Desarrollo Próximo (en el sentido propuesto por Vigotsky), en donde el aprendiz ya reconoce el problema a resolver, pero aún no es capaz de resolverlo por sí solo. Así pues, por medio de un andamiaje, el tutor ayuda eficazmente al aprendiz a resolver la tarea.

Ahora bien, existen diversas labores que el tutor debe afrontar en una tutoría, así como diversos niveles de ayuda que puede brindar, y diversas funciones de andamiaje que debe cumplir para que tal apoyo sea contingente. Según Wood, existen al menos tres dimensiones diferentes en una tutoría contingente. A saber, la contingencia instruccional (el cómo se apoya la actividad), la contingencia de dominio (el tema o contenido de la secuencia de tareas), y la contingencia temporal (el momento oportuno para intervenir). Estas dimensiones se exponen en la primera parte de la traducción de este artículo, la cual ha sido publicada en el número anterior de esta revista (Itinerario Educativo, Número 56, 2010).

En esta segunda parte de la traducción, el profesor Wood presenta sus ideas y reflexiones acerca de la interacción entre el 
estudiante y su ambiente de aprendizaje. En éstas, se abordan cuestiones bastante interesantes y pertinentes para cualquier educador o psicólogo que quiera comprender el comportamiento de sus educandos-aprendices. Se tratan preguntas tales como: ¿son los estudiantes que más ayuda necesitan los que más la solicitan? ¿Si se le brinda a un estudiante la oportunidad de recibir ayuda cuantas veces quiera, abusará de ésta para no esforzarse? ¿Saben los estudiantes que están en dificultades y que por ello deben pedir ayuda? ¿Son los estudiantes que resuelven más rápido una tarea los que más están aprendiendo? ¿Pueden los estudiantes de bajo rendimiento aprender a regular su propio ambiente de aprendizaje?

Si usted desea conocer la manera como el profesor David Wood aborda estos interrogantes y propone posibles respuestas, lo invitamos a que lea esta segunda entrega del artículo "El qué, por qué, cómo y cuándo de la tutoría: desarrollo de la ayuda y las habilidades de tutoría en niños".

Los traductores Luis Alejandro Andrade Lotero María Fernanda Cobo Charry

El qué, por qué, cómo y cuándo de la tutoría: desarrollo de la ayuda y las habilidades de tutoría en niños

\section{Se necesitan dos para realizar una tutoría}

La siguiente lista de enunciados delimita los temas en los que nos concentraremos para tratar de develar la forma en la que las diferencias individuales de los aprendices influyen en el establecimiento de las contingencias que nosotros observamos en las 
interacciones entre el tutor y el aprendiz. Los enunciados son los siguientes:

- Cómo regulan los niños su propia actividad y la del tutor.

- Cómo reaccionan los niños frente a una situación imprevista.

- Habilidad o disposición de los niños para buscar ayuda.

- Inatención aparente hacia la ayuda ofrecida.

- Dependencia excesiva de la ayuda.

- Asignación de tiempo en la tarea; acelerar o desacelerar con respecto a la experticia en la tarea a realizar.

Considero que puede ser muy útil empezar hablando acerca de la clase de predicciones y expectativas que motivaron nuestro trabajo, y cómo estas crecieron a partir de la teoría de la contingencia y el andamiaje.

Han habido cerca de 20 estudios diferentes sobre el andamiaje y la tutoría contingente en una gran variedad de contextos (ver por ejemplo Elber, 1996). Estos estudios han brindado una gran cantidad de evidencia empírica. No obstante, también han criticado el hecho de que, por una parte, existe una tendencia a prestar demasiada atención al tutor (quien, en mi experiencia, es demasiado negligente frente al aprendizaje) y por otra, que el andamiaje y la tutoría contingente conducen al niño a un rol pasivo. En lo que a mí concierne, nada está más lejos de ser verdad. Lo explicaré mediante un experimento mental.

Imaginemos que hay un tutor perfectamente contingente que está interactuando con dos aprendices. Uno de los niños tiene una alta aptitud para el aprendizaje en el dominio de conocimiento de la 
tarea presentada por el tutor. El otro niño, por su parte, debe esforzarse bastante para aprender. Dado que el tutor brinda una ayuda perfectamente contingente, uno podría predecir las dificultades relativas que enfrentan los dos niños solamente observando al tutor. El tutor contingente, en un sentido figurado, envuelve a los aprendices. El tutor los rodea complementando las necesidades del aprendiz para que sus actividades sean exitosas ${ }^{1}$. Así pues, yo creo que las personas se equivocan cuando dicen que esto lleva a que el aprendiz asuma un rol pasivo. No se puede tener una tutoría contingente a menos que se involucre al niño en una interacción colaborativa. De este modo, el rol del tutor contingente llevará el ritmo del progreso y de la actividad del aprendiz.

Pienso que hay un aspecto profundo en el cual la crítica mencionada tiene alguna validez. A saber, nosotros nunca estudiamos en detalle las diferencias individuales entre los niños en nuestros primeros trabajos (como hemos señalado en varias de nuestras publicaciones). Siempre hay niños que, aún lejos de una contingencia óptima de aprendizaje, hicieron extraordinarios progresos de aprendizaje. Algunos niños aprenden con sólo un poco de ayuda, tal vez porque están bendecidos con una alta aptitud para el aprendizaje. No obstante, otros chicos tienen que esforzarse mucho para poder aprender.

Esto último también se relaciona con otra crítica, la cual radica en que la tutoría contingente no se presenta frecuentemente en la vida cotidiana de los niños. Considero que aquello es un comentario perfectamente válido. La tutoría contingente es una descripción de un ideal casi imposible de lograr en la práctica, porque la complejidad y las demandas intelectuales sobre los tutores son inmensas. La tutoría contingente se convierte en un asunto serio

1 Para conocer más acerca de las funciones y actividades del tutor en una tutoría contingente, ver la primera parte de la traducción, publicada en el número 56 de esta revista. 
cuando se trata con aprendices que necesitan esforzarse mucho. Por supuesto, esto involucra a cualquiera de nosotros en algún momento de la vida o en ciertos casos. De hecho, uno de nuestros estudios, del cual hablaré más adelante, se basa en un área que muchos de nosotros encontramos difícil: polinomios en matemáticas. Escogimos este tema porque sabemos que la mayoría de las personas lo encuentran difícil de aprender, aún siendo inteligentes y brillantes.

Así pues, acepto que es una crítica justa el hecho de que una tutoría altamente contingente en el mundo que nos rodea no es fácil de encontrar. Esta es realmente un ideal, el cual adquiere más importancia tratándose de situaciones de difícil aprendizaje.

Ahora bien, para responder a la crítica hecha hacia la influencia que ejercen los aprendices en el proceso de tutoría, hablaré del estudio de la búsqueda de ayuda por parte de los aprendices. Tendremos bastante que decir al respecto. Investigadores en diversas partes del mundo han identificado diferencias individuales en niños que piden ayuda -típicamente piden ayuda a un adulto-, encontrándose hallazgos generales en la literatura académica (para un resumen de éstos, véase Wood \& Wood, 1999). En general, los niños que saben menos y que tienen que esforzarse mucho para aprender son, en promedio, menos hábiles para buscar ayuda. Ellos son menos dados a pedir ayuda cuando sus actividades sugieren que están en problemas, y ellos también parecen menos capaces de hacer uso de la ayuda que se les ha brindado. Este aspecto de la solicitud de ayuda no es de ninguna manera obvio, y, en general, va en contra de muchas de nuestras intuiciones.

Aquello es una paradoja cruel: los aprendices que necesitan más tutoría contingente son menos dados a llamar la atención del tutor, y también son menos capaces de beneficiarse de ésta cuando se les brinda. ¿̇Son los niños quienes más necesitan ayuda los menos capaces de establecer la relación tutorial? ¿Están ellos experi- 
mentando un doble infortunio? ¿Existirá un aspecto secundario al interior de las dificultades de aprendizaje, el cual se refiere a que los niños no piden ayuda a su tutor? Estas preguntas me llevaron a pensar en el título "Se necesitan dos para realizar una tutoría" y a intentar construir una imagen completa del papel del aprendiz en la formación de una relación tutorial.

¿De qué otras formas pueden los aprendices influenciar la manera como los tutores realizan la tutoría? En el video de los dos niños que resuelven una tarea de ir de compras ${ }^{2}$, el niño que inicialmente no supo cómo contar desde la moneda de diez, parecía alegre y dispuesto a recibir ayudar. Allí vimos un ejemplo de una excelente tutoría, pero lo cierto es que en ese contexto se encontraba un aprendiz fácil de llevar y bastante activo. Una de las características que hacen a este niño relativamente fácil de enseñar, sugiero yo, es su temperamento. Hay suficiente evidencia ahora de que los tipos extremos de temperamento tienen bases biológicas (Wood, 1998). Hay un estudio excelente realizado por Bell y Waldrop realizado en los Estados Unidos hacia los años de 1970 y comienzos de 1980, en el cual los investigadores identificaron dos grupos de niños que representan dos extremos de un continuo (la mayoría de nosotros nos encontramos en la mitad). En un extremo se hallaban los niños con un lento proceso de concentración. Estos niños presentaron bajos niveles de motivación y de concentración en situaciones de interacción sostenida. Tal vez estaban destinados a volverse los niños tímidos del futuro. El otro grupo era el de los niños impulsivos. Lo que me gusta del estudio de Bell y Waldrop es la manera como demostraron que la posición de un individuo en este continuo se encuentra asociada con la incidencia de anormalidades físicas menores. Es decir, esto último hace referencia a cosas tales como tener un dedo del pie más largo que el otro o alguna leve anormalidad en la forma de la lengua. Es improbable que cualquier asociación entre estas características físicas y el

2 Ver la primera parte de la traducción, publicada en el número 56 de esta revista. 
temperamento sean creados por las reacciones de otras personas hacia los niños porque son en gran medida invisibles. Este trabajo provee la evidencia más reveladora que conozco acerca de la relación entre el temperamento de los niños y predisposiciones biológicas. Lo que nadie ha hecho es explorar cuál es el impacto de estas características sobre la interacción social que se tendrá en momentos posteriores de la vida, como por ejemplo, una tutoría. Mi apuesta es que hay fuertes relaciones entre las estas dos variables (anormalidades físicas menores y temperamento -desatención e impulsividad), lo cual nos brinda una manera de explorar el impacto del niño sobre el tutor.

Cuando somos confrontados por los niños que son difíciles de enseñar, me pregunto si será particularmente arduo ayudarles a que aprendan estrategias de auto-regulación de su propio aprendizaje (como una forma de aprendizaje autodidacta). En otras palabras, ¿será que los niños a quienes es difícil enseñarles, encuentran igualmente difícil enseñarse a sí mismos? Pienso que probablemente hay alguna clase de asociación más específica entre niños que son difíciles de enseñar y niños que encuentran difícil regular su propio aprendizaje. De hecho, una de las razones por las que estoy tan interesado en el programa educativo Reading Recovery es porque este trata de ayudar explícitamente a los niños a que se vuelvan aprendices más eficientes. Este programa provee un contexto natural para averiguar el grado en el que se puede aprender a aprender a través de una tutoría. Considero que es muy importante que los niños aprendan la forma de regular su propio aprendizaje. Si esto es así, cualquier dificultad en el aprendizaje a través de una relación tutorial puede tener efectos muy generales sobre su futuro aprendizaje. Sospecho, sin embargo, que esto no es verdad para todos los niños, sino que lo será para aquellos niños que son difíciles de enseñar y que necesariamente enfrentarán problemas en aprender cómo regular su propio aprendizaje. Personalmente no creo que yo haya sido un niño fácil de enseñar, por ejemplo. No obstante, creo que hago un trabajo razonablemente bien al enseñarme a mí mismo. 
Así pues, aquí se encuentra otro grupo de preguntas que ayudaron a motivar el diseño y análisis de los estudios de los que hablaré a continuación. De nuevo, subrayo el hecho de que se trata de estudios realizados sobre ambientes de aprendizaje asistidos por computador, y reconozco que es peligroso generalizar los resultados sobre el fenómeno de buscar ayuda, la auto-regulación y el aprendizaje en entornos cara a cara. En efecto, aún no he explorado la relación existente entre la forma en la que los niños regulan su ambiente de aprendizaje cuando interactúan con un tutor mecánico y cómo regulan su proceso de aprendizaje cuando interactúan con profesores. De hecho, pienso que viendo el asunto en detalle, sería extremadamente difícil estudiar las interacciones establecidas cara a cara entre el niño y su tutor con la misma profundidad con la que es posible estudiar la relación tutorial con un computador, ya que en este último automáticamente se registran y almacenan muchos datos acerca de la interacción. En este sentido, puedo especular acerca de las implicaciones que los estudios basados en computador tienen para ayudarnos a entender la tutoría humana. Así pues, aceptaré sus opiniones respecto a si las generalizaciones que se van a presentar son acertadas o no.

\section{Buscando ayuda cuando uno está en problemas}

Un aspecto crucial para explorar hace referencia a la forma en que los aprendices reaccionan cuando se enfrentan a un impase, un error o una dificultad. Esto puede parecer un tema evidente, puesto que si los niños buscan ayuda activamente cuando no saben cómo proceder, podrían ayudar al tutor a construir un ambiente de aprendizaje contingente. En los tutores computacionales que hemos desarrollado, hemos dejado que sea el niño quien decida cuándo buscar ayuda. Así pues, la contingencia temporal (decisiones acerca de si intervenir o no y cuándo intervenir) se deja en manos de los niños. La contingencia instruccional sigue siendo asunto del tutor. El niño decide cuándo desea recibir ayuda, mientras que el tutor usa un registro del desempeño reciente de 
ese niño para decidir qué tanta ayuda necesita. Sobre la base de la respuesta del niño hacia la ayuda recibida, el tutor ofrece un nivel mayor de ayuda o comienza a desvanecerse. De esta manera pudimos comenzar a entender cómo niños con características diferentes buscan ayuda cuando sienten que se encuentran ante un impase o un error.

Cuando comenzamos el diseño del estudio de la contingencia temporal, estuvimos hablando con John Anderson, quien, con sus colegas en la Universidad de Carnegie Mellon, había realizado un trabajo pionero acerca de la tutoría basada en computador. Él había advertido sobre la precaución que había que tener al dejar la búsqueda de ayuda en manos de los aprendices. Porque, como él sospechaba, estos podrían abusar de la ayuda usándola en exceso para evitar esforzarse. En otras palabras, los aprendices manipularían al tutor para que les diera todas las respuestas, en vez de intentar resolver los problemas por sí mismos. Ahora bien, yo tengo una perspectiva diferente y tal vez más benigna sobre los aprendices. Yo considero que si un aprendiz necesita y recibe una ayuda de Nivel $3^{3}$, éste naturalmente intentará resolver el problema y llegar a una respuesta. No creo que, por el contrario, el aprendiz vaya a intentar que el tutor le ofrezca una ayuda de Nivel $5^{4} y$, por ello, le dé la respuesta así no más. De esta manera, me pregunto si los niños son, en general, abusivos con la ayuda que reciben. $\dot{i}$ Esperaría usted que algunos o muchos niños eviten el esfuerzo y usen la ayuda en demasía?

A partir de nuestra pesquisa teórica sobre la búsqueda de ayuda, sospechábamos que más niños tenderían a rechazar la ayuda -ya fuera evitando buscarla o no apreciando la necesidad de pedirla.

3 Los niveles de ayuda e intervención son descritos en la primera parte de la traducción. En este caso, se refiere a una intervención verbal junto con una indicación no verbal.

4 El nivel 5 de ayuda hace referencia a la modelación o demostración de la solución del problema. Ver. primera parte de la traducción. 
Así mismo, pensábamos que la mayoría de los que la rechazarían, serían aquellos que presentaban mayor necesidad de recibirla.

Un segundo asunto a explorar corresponde a si algunos niños son más difíciles de tutoriar contingentemente, porque no pueden o no entienden la ayuda recibida. Así pues, nos hicimos la siguiente pregunta: ¿Son todos los niños igualmente capaces de hacer uso efectivo de la ayuda cuando la piden o les es ofrecida? Por supuesto, siempre existe la posibilidad de que el tutor dé una ayuda inapropiada, pero este es un punto que trataremos más adelante.

\section{El uso del tiempo por parte de los aprendices en una tarea}

Considero que la tutoría basada en computador puede ofrecernos un panorama único desde donde podemos revisar un viejo tema, a saber, el de la forma en que los estudiantes usan su tiempo en una tarea. Como es sabido, hay suficiente evidencia que muestra que uno de los mejores puntos para predecir los logros de un aprendiz es el tiempo que éste dedica en una tarea. De hecho, el tiempo dedicado a una tarea nos invita a estudiar la eficacia de diferentes regímenes tutoriales. Un régimen tutorial que logre que el estudiante permanezca la mayor cantidad de tiempo posible en una tarea, probablemente contribuirá a mejorar el aprendizaje. Es difícil determinar cuáles son los efectos diferenciales de distintas estrategias de tutoría. Así mismo, es difícil conocer el impacto de detalles específicos de las estrategias de tutoría que apoyan el aprendizaje. Intentaré demostrar cómo los registros basados en computador de la actividad entre un tutor y un aprendiz pueden ser usados para estudiar estos asuntos importantes (aunque, como mencioné antes, podría ser peligroso generalizar los hallazgos encontrados a la tutoría realizada cara a cara).

Las preguntas acerca de qué tan estratégico es el aprendiz frente al uso de su tiempo en una tarea nos permite estudiar cómo el 
niño regula el ambiente tutorial -por ejemplo, trabajando rápido cuando siente que sabe cómo resolverla, pero trabajando despacio cuando está inseguro de qué hacer. Me sorprendió mucho los registros del programa Reading Recovery, en donde se puede apreciar la manera como se utiliza el tiempo con el aprendiz. Algunas veces el tutor parece tratar de acelerar el paso o acelerar la actividad del niño. En otras ocasiones, el tutor le deja mucho más tiempo al niño. Sospecho que los cambios en el ritmo obedecen a la evaluación que se ha hecho acerca del nivel de experticia de cada niño. Si uno percibe que éste ha entendido los aspectos básicos de una tarea, uno comienza a esperar que la tarea fluya más rápidamente. Por el contrario, si se espera que el niño aprenda nuevas cosas, se provee más tiempo y espacio para su realización. Así pues, el uso del tiempo refleja nuestras suposiciones acerca del punto en el que se encuentra el aprendiz.

Antes en esta exposición, utilicé la metáfora del ballet para describir las interacciones entre el tutor y los aprendices. Queremos saber ahora si, por ejemplo, algunos niños espontáneamente usan su tiempo para acelerar el paso en tareas que parecen familiares, pero reducen su ritmo cuando están inseguros. Me pregunto si los aprendices pueden ayudar al tutor a regular el uso del tiempo en una lección. ¿Serán otros aprendices menos efectivos en su manera de ayudar al tutor a construir un ambiente de aprendizaje contingente? Pienso que no hemos sido capaces de enfrentar estas preguntas antes, porque no habíamos podido registrar en un nivel de detalle minucioso las actividades de los aprendices. Los estudios basados en computador sí nos permiten resolver estas cuestiones. Aún más importante, una vez que dispongamos de esta información, podremos preguntarnos si el uso del tiempo en una tarea por parte de un niño se relaciona con sus resultados de aprendizaje. Y para adelantar la respuesta a esta pregunta, en efecto, es posible decir que el uso del tiempo se relaciona con los resultados de aprendizaje. 


\section{Zona de desarrollo próximo y búsqueda de ayuda}

Frecuentemente, las interacciones tutoriales efectivas se promueven trabajando con aquellas brechas que hay entre lo que el niño ha comenzado a reconocer, pero que aún no puede realizar por sí mismo. Los estudiantes pueden reconocer qué es lo que hay que hacer incluso antes de poder realizarlo ellos mismos. En este sentido, me parece que esta es una de las condiciones de la tutoría, y se trata de mantener al niño en tal punto que pueda reconocer soluciones que aún no puede resolver por sí solo. Por lo tanto, el niño es un jugador crítico en el proceso de aprendizaje, porque puede juzgar los resultados de sus propios esfuerzos.

La información que hemos recogido de nuestros tutores, sugiere que nuestro punto de vista es plausible. Y si es verdad, es de gran importancia porque nos acercamos a definir lo que es la tutoría contingente, aquella en la que se da una inducción acertada a la tarea, y aquella en la que existe un punto de vista compartido por el tutor y el aprendiz -elementos cruciales del andamiaje. Si la instrucción dada por el tutor es al mismo tiempo temporal e instruccionalmente contingente, nos acercamos al proceso tutorial ideal. Espero que esto ayude a explicar la gran importancia que posee el reconocer la ZDP del aprendiz, su auto-percepción de que sabe qué hacer en la tarea, así como el uso que hace del tiempo para resolverlas.

Ahora bien, una de las estrategias disponibles cuando un aprendiz no sabe qué hacer (aunque no es la única) es buscar ayuda. Esto lo puede hacer leyendo, navegando en internet, o buscando en bases de datos. También puede hacerlo preguntándole a un amigo, a un colega o a un tutor. Uno busca información adicional en el entorno social. Se pueden usar diferentes estrategias, pero todas tienen que ver con reconocer la utilidad de incorporar ideas, información, perspectivas y consejos de otras personas del exterior. 
Me pregunto por qué en los colegios no se hace mayor énfasis para que los estudiantes aprendan a buscar ayuda, o si realmente estamos desarrollando habilidades y estrategias para la búsqueda de ayuda en niños escolares. Esta es una pregunta general que no pretende ser retórica. Más bien, tiene que ver con los consejos que damos a los niños sobre qué hacer cuando no tienen confianza acerca de lo que deben hacer.

\section{El Tutor Cuadrático}

He mencionado antes que uno de los estudios de tutoría computarizada realizado por nosotros, involucraba aprender acerca de expresiones polinómicas en matemáticas. No entraré en detalle aquí acerca de las funciones cuadráticas y cúbicas y de la manera como se buscó representar y enseñar estas por medio de un tutor computarizado. Sin embargo, escogimos este tema porque sabíamos que los estudiantes de entre 11 y 13 años de edad aún no han sido formalmente introducidos a estos conceptos en el currículo. También sospechábamos que sólo alrededor de 10 o 15\% de los estudiantes llega a desarrollar una comprensión real acerca de este tema (estas estadísticas se basan en trabajos previos realizados por el grupo Chealsea Maths en el Reino Unido). Entonces, queríamos explorar la tutoría contingente en un área que era todo un desafío para cualquier persona. De esta manera pudimos probar nuestra teoría al límite.

Deliberadamente escogimos estudiantes dentro de un amplio espectro de desempeño para que trabajaran con el tutor computarizado. Recordemos que el tutor ofrece instrucción contingente pero sólo cuando el estudiante la solicita. Las decisiones acerca de cuándo buscar ayuda y de muchos aspectos del ritmo y el paso de las sesiones tutoriales se encontraban en manos de los estudiantes. La tasa de éxito (cuántos problemas resolvieron, cuánto tardaron, etc.) se debió, pues, exclusivamente a diferencias individuales de un aprendiz a otro. 
Si nuestro diseño tutorial era correcto, y nuestras predicciones provenientes de la teoría de la contingencia también, debería cumplirse que el tutor contingente se acomodaría al estudiante. De este modo, los diferentes patrones de interacciones entre diferentes aprendices y el tutor deberían reflejar diferencias individuales debidas a los aprendices. Se entiende que solamente al observar el comportamiento del tutor, se pueden hacer inferencias acerca de qué clase de niño se está tutoriando en un momento dado, es decir, niños hábiles o niños difíciles.

Aquí debo resaltar un aspecto crucial acerca de CUADRATIC, nuestro tutor computarizado. Aunque el tutor está diseñado para ser instruccionalmente contingente, no es contingente con respecto al conocimiento de dominio. En otras palabras, funciona como la enseñanza en el colegio, en las pruebas evaluativas o en los experimentos psicológicos, en el sentido de que confronta a todos los niños con la misma secuencia de problemas. Tal aspecto, como se puede ver, coloca una restricción importante sobre cómo deberían interpretarse los diferentes desempeños de los estudiantes de alto o bajo rendimiento.

Antes de ofrecerles tutoría, se evaluó a todos los niños para determinar sus conocimientos previos sobre razonamiento algebráico (ver Wood \& Wood). Después que los niños trabajaron con el tutor, efectuamos las usuales pruebas de salida y se evaluó el nivel de retención de conocimientos a largo plazo. Se trataba de un clásico diseño experimental de la forma pre-test, intervención, y pos-test.

Si nuestras expectativas acerca de la tutoría contingente iban a ser confirmadas, deberíamos encontrar que las diferencias individuales de los puntajes del pre-test se correlacionaban altamente con las interacciones medidas con el tutor. En efecto, así fue. Tomando todos los resultados juntos, los datos on-line de CUADRATICO produjeron múltiples correlaciones con los puntajes de las evaluaciones $(c=0,8)$. En efecto, argumentaré 
que el tutor está midiendo las mismas diferencias individuales que produjo la prueba de entrada, pero por una ruta diferente. No sorprenderá saber que los aprendices que en la prueba de entrada reflejaron una más alta aptitud para las matemáticas, trabajaron a una tasa más rápida con el tutor CUADRATICO, hicieron menos movimientos incorrectos y buscaron ayuda en menos ocasiones. Es necesario notar, sin embargo, que es posible que hayan buscado menos ayuda debido a que experimentaron menos dificultades. Estos estudiantes también tendieron a autocorregirse. Es importante tener en cuenta que CUADRATICO nunca forzó la ayuda al estudiante. El tutor únicamente decía "Sí" o "No" a las acciones correctas o incorrectas, pero los estudiantes tuvieron el $100 \%$ de oportunidad para auto-corregirse, y tanto tiempo como quisieron para trabajar en la prueba. Así pues, cuanto más conocimiento tenía el estudiante, más probable era que se auto-corrigiera.

También se encontró que era más probable que los estudiantes con mejores rendimientos supieran cuándo buscar ayuda. Esta afirmación surge de examinar cuántas veces cada estudiante buscó ayuda luego de fallar una respuesta. Los puntajes más altos reportaron que estos estudiantes tuvieron menos errores y que buscaron ayuda menos frecuentemente en promedio, pero la probabilidad de que buscaran ayuda luego de cometer una equivocación fue más alta. Dicho de otra manera, los puntajes más bajos fueron quienes tendían a producir toda una secuencia de errores encadenados, a pesar del hecho de que el sistema les había dado retroalimentación acerca de sus errores. Así pues, los puntajes altos pudieron auto-corregirse más, y si no lo hacían, había más probabilidad de que pidieran ayuda, con la consecuencia de presentar menos errores.

Estos resultados corroboraron las malas noticias acerca de los niños a quienes se les dificulta aprender. Yo estoy de acuerdo con John Anderson respecto a su conclusión de que los errores frecuen- 
temente impiden el aprendizaje. Nuestros hallazgos con DATA concuerdan con otros tantos que han encontrado correlaciones negativas entre las tasas de error y los resultados de aprendizaje. Los errores no solamente conllevan más tiempo, sino que también parecen restar la motivación para el aprendizaje. Ahora bien, yo no soy un skinneriano y no quiero llevar este argumento muy lejos. Si uno recuerda, Fred Skinner tenía la ambición de producir condiciones de aprendizaje sin errores y diseñó sus primeras máquinas con este objetivo en mente. No obstante, considero que no todos los errores son del mismo tipo, y que algunos de ellos pueden ser importantes para el camino hacia el aprendizaje. Los errores están, por decirlo de alguna manera, en nuestra mente y no en la mente del niño -quien simplemente podría querer ensayar a ver qué pasa, por ejemplo, y no sólo resolver el problema. De esta manera, cuando uso aquí el término errores, este debe ser leído con comillas. De este modo, los errores pueden ser un producto normal e importante del proceso de aprendizaje. Por ejemplo, cuando un niño está explorando, éste comete errores desde el punto de vista del tutor, pero aquello que busca puede ser importante de descubrir para entender la naturaleza de las posibilidades de una situación dada.

No obstante, la evidencia encontrada sí muestra que es la persistencia del error de cara a la retroalimentación, es decir, luego de recibir ayuda, lo que inhibe el aprendizaje. Algunos de los niños del experimento produjeron hasta 30 intentos sin éxito, uno tras otro, probablemente adivinando. Con todo, estos niños se hallaban enfrentados a una clase de problemas matemáticos que muy probablemente no podían resolver por sí solos.

Una implicación es que estos niños son más difíciles de tutoriar debido a que dan pocas y menos claras señales de que necesitan ayuda. Ciertamente, ayudan menos al tutor al no auto-corregirse, lo cual es una pieza vital dentro del proceso de aprendizaje. La auto-corrección es un claro signo de que el niño ha reconocido la 
importancia de los efectos de sus propias acciones - una forma de metacognición o aprendizaje de su propia actividad cognitiva. La auto-corrección es un signo real de auto-regulación estratégica en progreso.

Asimismo, los resultados también mostraron que aquellos niños a quienes más se les dificultaron las tareas tutoriales con CUADRATICO, tuvieron más probabilidades de haber obtenido un puntaje bajo en las pruebas de entrada. Me pregunto entonces si tener una mala estrategia para regular el ambiente de aprendizaje es una de las razones que explica el por qué estos estudiantes tienen un bajo rendimiento. Es decir, ¿̇será posible que estemos comenzando a entender el proceso de por qué estos niños presentan un bajo rendimiento, es decir, en tanto no son buenos al regular su propio ambiente de aprendizaje? Esta es una buena pregunta, la cual intentaré responder más adelante.

\section{¿Trabajar rápido es un signo de aprendizaje efectivo?}

Los niños que saben más, como hemos visto, trabajan más rápido, son más precisos, son más autónomos, y obtienen mejores tasas de ganancia en aquellos aspectos en los que son tutoriados. Cuanto más sepa uno desde el comienzo, más probable es que aprenda más; aún cuando se haya dado un tiempo igual para aprender que a otro niño que sabe menos. En este sentido, ¿̇será posible concluir que un mejor aprendizaje es rápido, sin errores y autónomo? ¿Será esto lo que deseamos que los niños exhiban como comportamiento? Ciertamente, a partir de mi propia experiencia con ambientes de aprendizaje (y juegos) computarizados disponibles comercialmente, parece que promocionan y recompensan un desempeño veloz y libre de errores. No obstante, ¿̇qué pudimos concluir nosotros a partir de nuestros resultados acerca de que resolver problemas de manera rápida, sin errores y autónomamente, puede ser tomado como un signo de un mejor aprendizaje? 
Nosotros encontramos que no. Posiblemente también encontramos una asociación entre la magnitud de los logros de aprendizaje y el desempeño con el tutor, ya que los estudiantes de alto rendimiento trabajaron más rápido y también aprendieron más. No obstante, no encontramos que trabajar rápido causara un mejor aprendizaje. De hecho, pudimos simplemente estar midiendo diferencias individuales en conocimiento previo por medio de una serie de medidas distintas -velocidad, auto-corrección, autonomía, etc. Intentaré explicar esto desde otro ángulo.

Imaginemos que hay dos niños con puntajes exactamente iguales en una prueba de entrada. Estos dos niños van y trabajan con el tutor, y uno de ellos aprende más que el otro. ¿̇Fue este niño el que trabajó más rápido o el que trabajó más despacio? De hecho, es más probable que sea el que trabajó más despacio de los dos. Estadísticamente es posible evaluar esta hipótesis si se toma todo el grupo de estudiantes y se compara la asociación entre el conocimiento previo (medido en la prueba de entrada) y los resultados de aprendizaje (medidos en la prueba de salida) con la interacción con el tutor y los resultados de aprendizaje. Al hacer esto, encontramos que los mejores resultados de aprendizaje estuvieron generalmente asociados a una tasa de trabajo lento. Así pues, nos gustaría sugerir a aquellos niños que encuentran dificultades en su aprendizaje, que resuelvan los problemas despacio, y que no se apuren.

No creo que antes se haya podido investigar esto con tanto detalle como ahora: la relación que hay entre conocimiento previo, aprendizaje y tutoría, y resultados de aprendizaje, todos relacionados entre sí. Esta es una de las ventajas de trabajar con los sistemas basados en computador. Sin embargo, no puedo decir si estos aprendizajes son o no verdad para la tutoría cara a cara. Tendré que dejar al lector para que realice sus propias inferencias sin ninguna evidencia útil. No obstante, sospecho que es posible que podamos estar de acuerdo en que la relación existente entre los 
resultados de aprendizaje y el ritmo de aprendizaje en una tutoría es un aspecto crucial.

Por otro lado, los aprendices que aprendieron más (luego de haber descartado las diferencias individuales en las pruebas de entrada y de salida) no solamente trabajaron más despacio con el tutor, sino que también evitaron quedar bloqueados frente a un impase, y evitaron el error al buscar ayuda. El impacto de haber buscado ayuda antes que arriesgarse a una serie de errores consecutivos fue, de hecho, significativo para los estudiantes de bajo rendimiento. Hubo evidencia real de que buscar ayuda fue incluso más importante para aquellos a quienes se les dificultaba aprender. También fue importante para nosotros como diseñadores de la tutoría porque nos mostró que nuestra ayuda logró ayudar teniendo un efecto benéfico en el aprendizaje. Se dedujo, así mismo, que los niños no podían haber estado abusando de la ayuda computarizada. Si lo hubieran estado haciendo no esperaríamos haber encontrado ninguna relación (o más bien una relación negativa) con los resultados de aprendizaje, ya que habrían estado usando el tutor para evitar esforzarse en su aprendizaje, esto es, habrían estado haciendo trampa. Fue tal como había sospechado: tan pronto como el estudiante tuvo suficientes pistas para resolver el problema, se dedicó a resolverlo.

Finalmente, expondré brevemente algunas reflexiones acerca del uso del tiempo por parte de los niños. Vamos a ver si se corresponden a las intuiciones que usted pueda tener hasta este momento. No es de sorprender que los niños de alto rendimiento respondan más rápidamente que los de bajo rendimiento. ¿̇Implica esto que los de alto rendimiento se están dando a sí mismos más tiempo para responder? ¿Qué pasa entonces con las respuestas incorrectas? ¿Tienden estas a ser dadas más rápido o más despacio? De hecho, las respuestas incorrectas fueron dadas más rápido, es decir, el tiempo entre la respuesta anterior y la respuesta nueva (errada), fue más corto. En efecto, para más del 90\% de los niños, 
el tiempo de respuesta para los errores fue más corto que para las respuestas correctas, y los de alto rendimiento cometieron errores con tiempo de respuesta más corto que los de bajo rendimiento. Esto es bastante intrigante. ¿̇Significa que los errores provienen de un comportamiento impulsivo? Entonces, ¿̇son los niños de alto rendimiento más impulsivos, o, luego de haber encontrado rápidamente que no sabían responder a la pregunta, se inclinaron por la estrategia de ensayo y error? No lo puedo decir, aunque me inclino a que sea más plausible esta última explicación.

Sin embargo, más importante es el hecho de que el tiempo promedio para buscar ayuda con CUADRATICO fue más largo que el tiempo tanto de los errores como de los aciertos. Esto implica que los niños estaban pensando antes de decidirse a buscar ayuda. Los aprendices estaban generalmente tomándose su tiempo para pensar acerca del problema antes de solicitar ayuda. Si usted está de acuerdo, esto implica que, como tutor, es necesario preguntarse a sí mismo si se deja el tiempo suficiente a los niños para que decidan a buscar ayuda antes que ofrecerla personalmente. Además, sería bueno reflexionar si uno deja el tiempo suficiente para que el niño intente auto-corregirse después de un error.

\section{DATA: ćson los estudiantes de bajo rendimiento generalmente menos eficaces regulando su propio ambiente de aprendizaje?}

Recientemente hemos completado una serie de estudios usando otro tutor contingente, el cual fue denominado DATA por sus siglas en inglés Dynamic Assessment and Tutoring in Arithmetic (Evaluación dinámica y tutorial en aritmética). Explicaremos lo sucedido con este tutor para estudiar la contingencia de dominio. DATA fue diseñado para evaluar el desarrollo de cada aprendiz en el dominio de conceptos básicos como el de número, adición y sustracción. Después de una detallada evaluación en línea, el tutor es contingente con el patrón de dominio en la 
tarea de cada niño. Este ofrece experiencias para la resolución de problemas que son presentados a la medida de cada niño, según su desempeño. DATA siempre comienza desde los problemas más simples en los que cada niño tuvo alguna dificultad, y desde allí comienza a presentar problemas más avanzados sobre la base de su desempeño con el tutor.

Si DATA tiene éxito en conseguir un grado de contingencia de dominio, entonces deberíamos encontrar que los niveles de dificultad relativos a los problemas brindados por el tutor, crean un mapa de diferencias individuales en cuanto a los logros de los estudiantes en matemáticas. Hemos puesto a prueba al tutor extensivamente, y tenemos confianza de que este sea el caso. Así pues, tenemos alguna evidencia de que es posible diseñar principios de tutoría contingente de dominio: un tutor que se adapta al aprendiz al encontrar tareas tutoriales que se ajustan a sus niveles actuales de conocimiento y de rendimiento.

Pero la razón principal para hablar de DATA en esta presentación son algunos hallazgos sorprendentes acerca de la búsqueda de ayuda, autocorrección y el uso del tiempo en una tarea por parte de estudiantes de alto y bajo rendimiento. Básicamente, cuando uno observa estas características de desempeño de los niños al usar DATA, no se encuentran diferencias asociadas a los rendimientos previos. Los puntajes bajos tenían tanta probabilidad como sus pares de puntaje alto de buscar ayuda, autocorregirse y de trabajar a un ritmo lento o rápido con el tutor. Los niños que trabajaron más despacio con el tutor, como en QUADRATIC, tendieron a conseguir más éxito en la resolución de problemas. Así mismo, la tasa de trabajo con los problemas del tutor fue bastante independiente en relación a los puntajes obtenidos en la prueba de entrada.

Estos hallazgos, los cuales no habíamos esperado, serían bastante importantes si pudieran ser generalizados. Indicarían que los estudiantes de bajo rendimiento no son, como habíamos pensado, 
generalmente menos eficaces para regular al tutor o a su propio aprendizaje.

Cuando uno observa la literatura de la interacción cara a cara y la búsqueda de ayuda, todos señalan en una misma dirección: los estudiantes de bajo desempeño tienden a ser menos eficaces que los estudiantes de alto desempeño frente a la auto-regulación -particularmente en la búsqueda de ayuda y de explicación. Esto conduce a mantener la idea general y ampliamente aceptada de que la autorregulación juega un papel importante de tales diferencias individuales. Nuestros hallazgos a partir de DATA ponen en entredicho esta idea. ¿̇Por qué son nuestros hallazgos tan diferentes a los encontrados por otros estudios en esta área?

En todos los estudios de la literatura que yo he buscado, nadie ha hecho honor a las demandas de la contingencia de dominio. Y es por ello que nosotros realizamos tales experimentos. Usualmente, cuando se realizan experimentos, uno desea medir y comparar con el mismo rasero, $y$, de esta manera, brindarles a todos los participantes la oportunidad de que resuelvan la misma tarea. De hecho, si uno no se asegura de que todos los participantes reciban el mismo tratamiento, los editores se podrían rehusar a publicar el estudio sobre la base de que los datos no son confiables. Necesitábamos algunas maneras de salirnos de esta situación si queríamos evitar que invalidaran las generalizaciones acerca del aprendizaje y la meta-cognición. Cuando se realiza un experimento o una situación de mediación, especialmente en contextos de enseñanza, todos los aprendices reciben los mismos problemas, similar clase de ayuda, y tal vez, similar cantidad de tiempo para resolver las tareas. Esto significa que los aprendices que saben menos al comenzar la prueba se enfrentan a problemas más difíciles que aquellos que saben más.

Si los estudiantes que saben menos exhiben un nivel inferior de búsqueda de ayuda, o tienen menos conciencia de su propio 
conocimiento y habilidades, ¿̇cómo deberíamos interpretar los siguientes problemas? ¿ Se deben tales diferencias a algo que está dentro de estos aprendices, en términos de estrategias menos eficaces de autorregulación? $\mathrm{O}$, ies solamente debido al hecho de que estos están más confundidos e inseguros respecto a los problemas que son más difíciles? ¿Será que experimentan el éxito con menos frecuencia y reciben ayuda que no es contingente frente a sus problemas reales? Todas estas posibles situaciones podrían presentarse si hace falta contingencia de dominio. Es posible entonces que DATA refleje una imagen diferente del aprendizaje de los niños, porque provee una situación única en la cual cada aprendiz es comparado con otros mientras trabajan en problemas que son contingentes respecto a niveles individuales en el dominio de la tarea.

En el programa Reading Recovery, los tutores intentan conseguir la contingencia de dominio todo el tiempo. Estos ajustan y adaptan las demandas en tareas de lectura y escritura para que se corresponda con los registros de desempeño y conocimiento de cada niño. No se trabaja a través de un único programa, o se expone a los niños a la misma secuencia de aprendizaje. El tutor ajusta y adapta las demandas que le pone al aprendiz en un esfuerzo de lograr un nivel de desafío que sea adecuado a cada uno. Me pregunto si, por ejemplo, cuando se ajustan los niveles de demanda en una situación de lectura y escritura a las necesidades del aprendiz, es posible encontrar conexiones obvias entre regulaciones efectivas de aprendizaje (búsqueda de ayuda, uso del tiempo en la tarea y auto-corrección) y los niveles de rendimiento previo de los niños. Por supuesto, los niños varían entre ellos con respecto a estos aspectos de auto-regulación. Pero, teniendo en cuenta los resultados encontrados con DATA, ¿̇serán tales diferencias individuales independientes de los niveles de rendimiento de los niños? Si esto es así, las implicaciones educativas y psicológicas serían realmente importantes. ¿Será posible que le estuviésemos atribuyendo habilidades de aprendizaje menos eficaces a ciertos 
niños, cuando realmente podrían ser debidas a nuestra falta de conocimiento acerca del qué, cuándo y cómo tutoriar?

\section{Agradecimientos}

Estoy realmente en deuda con Emily Rodgers quien se ofreció a transcribir esta charla sobre la que el presente documento se basa. Sin sus esfuerzos este artículo nunca hubiera aparecido. No espero se le atribuya la responsabilidad de este producto. Si alguien lo encuentra menos que satisfactorio, cualquier debilidad es enteramente mi responsabilidad. También agradezco al equipo de Atlanta quienes diseñaron el programa, hicieron funcionar el instituto, y me entrenaron en el programa tutorial Reading Recovery. También agradezco con gratitud la colaboración de mi esposa y colega Heather Wood quien se comprometió con la mayor parte del trabajo difícil sobre las partes teóricas y empíricas de este artículo en lo referente a la tutoría contingente. Finalmente, reconozco el apoyo financiero del consejo económico y social de investigación que financió CREDIT durante el periodo en el cual mucha de la investigación reportada fue realizada.

\section{Referencias}

DAWKINS, R. (1989). The selfish gene. New York: Oxford University Press.

ELBERS, E. (Ed.). (1996). Cooperation and social context in adultchild interaction. Learning and Instruccion. 6 (4), 281-286.

KOSLOWSKI, B. \& BRUNER, J. (1972). "Learning to use a lever". En: Child Development, 43 (3), 790-791.

MEAD, G.H. (1950). Mind, self and society. Chicago, IL: Chicago University Press.

NUNES, T., \& BRYANT, P. (1996). Children doing mathematics. Oxford, UK: Blackwell. 
WOOD, D. (1998). How Children Think and learn ( $2^{\text {nd }}$ ed.). Oxford, UK: Blackwell.

WOOD, D., BRUNER, J., \& ROSS, G. (1976). "The role of tutoring in problem solving". En: Journal of Child Psychology and psychiatry, 17, $89-100$.

WOOD, H. \& WOOD, D. (1999). "Help seeking, learning and contingent tutoring". En: Wood, D. (Ed.), Computers and Education, 33 (2/3), 153-170.

WOOD, D., WOOD, H., AINSWORTH, S., \& O'MALLEY, C. (1995).

"On becoming a tutor: Towards an ontogenetic model". En: Cognition and Instruction, 13 (4), 565-581. 